\title{
Approximation on the reciprocal functional equation in several variables in matrix non-Archimedean random normed spaces
}

\author{
Ali Ebadian' ${ }^{1}$, Somaye Zolfaghari', Saeed Ostadbashi' and Choonkil Park2*
}

\section{"Correspondence:}

baak@hanyang.ac.kr

${ }^{2}$ Research Institute for Natural

Sciences, Hanyang University, Seoul,

133-791, Republic of Korea

Full list of author information is

available at the end of the article

\begin{abstract}
In this paper, we investigate the generalized Hyers-Ulam stability of a reciprocal type functional equation in several variables in matrix non-Archimedean random normed spaces by direct and fixed point methods.

MSC: 39B52; 47H10; 46S50; 54E70; 39B72; 47H09

Keywords: matrix non-Archimedean random normed spaces; Rassias reciprocal functional equation; general reciprocal functional equations; adjoint and difference functional equations; fixed point alternative; Hyers-Ulam-Rassias stability
\end{abstract}

\section{Introduction}

About 70 years ago, Ulam [1] raised the well-known stability problem of functional equations. In the next year, Ulam's problem was partially answered by Hyers [2] in Banach spaces. Aoki [3] generalized Hyers' theorem for additive mappings in the year 1950. In the year 1978, a generalized version of the theorem of Hyers for approximately linear mappings was given by Rassias [4]. During 1982-1989, Rassias [5-7] treated the UlamGăvruta-Rassias stability on linear and non-linear mappings and generalized Hyers' result. In 1994, a further generalization of the Rassias theorem was obtained by Găvruta [8], who replaced the bound $\theta\left(\|x\|^{p}+\|y\|^{p}\right)$ by a general control function $\phi(x, y)$. The stability problems of several functional equations have been extensively investigated by a number of mathematicians, posed with creative thinking and critical dissent who have arrived at interesting results (see [8-18]).

In the year 2010, Ravi and Senthil Kumar [19] investigated the generalized Hyers-Ulam stability for the reciprocal functional equation

$$
r(x+y)=\frac{r(x) r(y)}{r(x)+r(y)}
$$

where $r: X \rightarrow Y$ is a mapping on the spaces of non-zero real numbers. The reciprocal function $r(x)=\frac{c}{x}$ is a solution of the functional equation (1.1).

(c) 2015 Ebadian et al. This article is distributed under the terms of the Creative Commons Attribution 4.0 International License (http://creativecommons.org/licenses/by/4.0/), which permits unrestricted use, distribution, and reproduction in any medium, provided you give appropriate credit to the original author(s) and the source, provide a link to the Creative Commons license, and indicate if changes were made. 
Later, Ravi et al. [20] introduced the reciprocal difference functional equation

$$
r\left(\frac{x+y}{2}\right)-r(x+y)=\frac{r(x) r(y)}{r(x)+r(y)}
$$

and the reciprocal adjoint functional equation

$$
r\left(\frac{x+y}{2}\right)+r(x+y)=\frac{3 r(x) r(y)}{r(x)+r(y)}
$$

and investigated the generalized Hyers-Ulam stability for the above two functional equations (1.2) and (1.3).

Recently, Ravi et al. [21] discussed the generalized Hyers-Ulam stability for the generalized reciprocal functional equation

$$
r\left(\sum_{i=1}^{m} \alpha_{i} x_{i}\right)=\frac{\prod_{i=1}^{m} r\left(x_{i}\right)}{\sum_{i=1}^{m}\left[\alpha_{i}\left(\prod_{\substack{j=1 \\ j \neq i}}^{m} r\left(x_{j}\right)\right)\right]}
$$

for arbitrary but fixed real numbers $\alpha_{i} \neq 0$, for $i=1,2, \ldots, m$, so that $0<\alpha=\alpha_{1}+\alpha_{2}+\cdots+$ $\alpha_{m}=\sum_{i=1}^{m} \alpha_{i} \neq 1$ and $r: X \rightarrow Y$ where $X$ and $Y$ are the sets of non-zero real numbers.

Very recently, Ravi et al. [22] obtained the general solution and investigated the generalized Hyers-Ulam stability of a reciprocal type functional equation in several variables of the form

$$
\frac{\prod_{\ell=2}^{m} r\left(x_{1}+x_{\ell}\right)}{\sum_{\ell=2}^{m}\left[\prod_{\substack{k=2 \\ k \neq \ell}}^{m} r\left(x_{1}+x_{k}\right)\right]}=\frac{\prod_{\ell=1}^{m} r\left(x_{\ell}\right)}{\sum_{\ell=2}^{m} r\left(x_{1}\right)\left[\prod_{\substack{k=2 \\ k \neq \ell}}^{m} r\left(x_{k}\right)\right]+(m-1) \prod_{\ell=2}^{m} r\left(x_{\ell}\right)},
$$

where $m$ is a positive integer with $m \geq 3$ in various normed spaces.

Remark 1.1 Ravi et al. [22-24] gave some counter-examples for the stability of reciprocal functional equations in singular cases.

In this paper, we apply a direct method and a fixed point method to investigate the generalized Hyers-Ulam stability of the functional equation (1.5) in matrix non-Archimedean random normed spaces.

\section{Preliminaries}

In this section, we recall some definitions and results which will be used later in the article.

A triangular norm (shorter $t$-norm) is a binary operation on the unit interval $[0,1]$, i.e., a function $T:[0,1] \times[0,1] \rightarrow[0,1]$ such that for all $a, b, c \in[0,1]$ the following four axioms satisfied:

(1) $T(a, b)=T(b, a)$ (commutativity);

(2) $T(a,(T(b, c)))=T(T(a, b), c)$ (associativity);

(3) $T(a, 1)=a$ (boundary condition);

(4) $T(a, b) \leq T(a, c)$ whenever $b \leq c$ (monotonicity).

Let $\mathcal{K}$ be a field. A non-Archimedean absolute value on $\mathcal{K}$ is a function $|\cdot|$ from $\mathcal{K}$ into $[0, \infty)$ such that for any $a, b \in \mathcal{K}$ we have 
(1) $|a| \geq 0$ and equality holds if and only if $a=0$;

(2) $|a b|=|a||b|$;

(3) $|a+b| \leq \max \{|a|,|b|\}$.

The condition (3) is called the strict triangle inequality. By (2), we have $|1|=|-1|=1$. Thus, by induction, it follows from (3) that $|n| \leq 1$ for each integer $n$. We always assume in addition that $|\cdot|$ is non-trivial, i.e., that there is an $a_{0} \in \mathcal{K}$ such that $\left|a_{0}\right| \neq 0,1$.

Let $X$ be a vector space over a scalar field $\mathcal{K}$ with a non-Archimedean non-trivial valuation $|\cdot|$. A function $\|\cdot\|: X \rightarrow \mathbb{R}$ is a non-Archimedean norm (valuation) if it satisfies the following conditions:

$\left(\mathrm{NA}_{1}\right)\|x\|=0$ if and only if $x=0$;

$\left(\mathrm{NA}_{2}\right) \quad\|r x\|=|r|\|x\|$ for all $r \in \mathcal{K}$ and $x \in X$;

$\left(\mathrm{NA}_{3}\right)\|x+y\| \leq \max \{\|x\|,\|y\|\}$ for all $x, y \in X$ (the strong triangle inequality).

Then $(X,\|\cdot\|)$ is called a non-Archimedean space.

Thanks to the inequality

$$
\left\|x_{m}-x_{l}\right\| \leq \max \left\{\left\|x_{j+1}-x_{J}\right\|: l \leq J \leq m-1\right\} \quad(m>l)
$$

a sequence $\left\{x_{m}\right\}$ is Cauchy if and only if $\left\{x_{m+1}-x_{m}\right\}$ converges to zero in a nonArchimedean space. By a complete non-Archimedean space we mean one in which every Cauchy sequence is convergent.

In 1897, Hensel [25] introduced a normed space, which does not have the Archimedean property.

In the sequel, we adopt the usual terminology, notations, and conventions of the theory of random normed spaces as in [26-29]. Throughout this paper, $\Delta^{+}$is the space of distribution functions, that is, the space of all mappings $F: \mathbb{R} \cup\{-\infty, \infty\} \rightarrow[0,1]$ such that $F$ is left-continuous and non-decreasing on $\mathbb{R}, F(0)=0$, and $F(+\infty)=1$. $\mathcal{D}^{+}$is a subset of $\Delta^{+}$consisting of all functions $F \in \Delta^{+}$for which $l^{-} F(+\infty)=1$, where $l^{-} f(x)$ denotes the left limit of the function $f$ at the point $x$, that is, $l^{-} f(x)=\lim _{t \rightarrow x^{-}} f(t)$. The space $\Delta^{+}$is partially ordered by the usual point-wise ordering of functions, i.e., $F \leq G$ if and only if $F(t) \leq G(t)$ for all $t$ in $\mathbb{R}$.

Definition 2.1 [29] A non-Archimedean random normed space (briefly, NA-RN-space) is a triple $(X, \mu, T)$, where $X$ is a vector space, $T$ is a continuous $t$-norm, and $\mu$ is a mapping from $X$ into $\mathcal{D}^{+}$such that the following conditions hold:

(RN1) $\mu_{x}(t)=\varepsilon_{0}(t)$ for all $t>0$ if and only if $x=0$;

(RN2) $\mu_{\alpha x}(t)=\mu_{x}\left(\frac{t}{|\alpha|}\right)$ for all $x \in X, \alpha \neq 0$;

(RN3) $\mu_{x+y}(\max \{t, s\}) \geq T\left(\mu_{x}(t), \mu_{y}(s)\right)$.

It is easy to see that if (RN3) holds, then we have

$$
\mu_{x+y}(t+s) \geq T\left(\mu_{x}(t), \mu_{y}(s)\right)
$$

Definition 2.2 Let $(X, \mu, T)$ be an NA-RN-space.

(1) A sequence $\left\{x_{n}\right\}$ in $X$ is said to be convergent to $x$ in $X$ if, for every $\epsilon>0$ and $\lambda>0$, there exists a positive integer $N$ such that $\mu_{x_{n}-x}(\epsilon)>1-\lambda$ whenever $n \geq N$. 
(2) A sequence $\left\{x_{n}\right\}$ in $X$ is called a Cauchy sequence if, for every $\epsilon>0$ and $\lambda>0$, there exists a positive integer $N$ such that $\mu_{x_{n}-x_{n+1}}(\epsilon)>1-\lambda$ whenever $n \geq N$.

(3) An NA-RN-space $(X, \mu, T)$ is said to be complete if and only if every Cauchy sequence in $X$ is convergent to a point in $X$.

We will also use the following notations. The set of all $m \times n$-matrices in $X$ will be denoted by $M_{m, n}(X)$. When $m=n$, the matrix $M_{m, n}(X)$ will be written as $M_{n}(X)$. The symbols $e_{j} \in M_{1, n}(\mathbb{C})$ will denote the row vector whose $j$ th component is 1 and the other components are 0 . Similarly, $E_{i j} \in M_{n}(\mathbb{C})$ will denote the $n \times n$ matrix whose $(i, j)$-component is 1 and the other components are 0 . The $n \times n$ matrix whose $(i, j)$-component is $x$ and the other components are 0 will be denoted by $E_{i j} \otimes x \in M_{n}(X)$.

Let $(X,\|\cdot\|)$ be a normed space. Note that $\left(X,\left\{\|\cdot\|_{n}\right\}\right)$ is a matrix normed space if and only if $\left(M_{n}(X),\|\cdot\|_{n}\right)$ is a normed space for each positive integer $n$ and $\|A x B\|_{k} \leq\|A\|\|B\|\|x\|_{n}$ holds for $A \in M_{k, n}, x=\left[x_{i j}\right] \in M_{n}(X)$ and $B \in M_{n, k}$, and that $\left(X,\left\{\|\cdot\|_{n}\right\}\right)$ is a matrix Banach space if and only if $X$ is a Banach space and $\left(X,\left\{\|\cdot\|_{n}\right\}\right)$ is a matrix normed space.

Let $E, F$ be vector spaces. For a given mapping $h: E \rightarrow F$ and a given positive integer $n$, define $h_{n}: M_{n}(E) \rightarrow M_{n}(F)$ by

$$
h_{n}\left(\left[x_{i j}\right]\right)=\left[h\left(x_{i j}\right)\right]
$$

for all $\left[x_{i j}\right] \in M_{n}(E)$.

We introduce the concept of matrix non-Archimedean random normed space.

Definition 2.3 Let $(X, \mu, T)$ be a non-Archimedean random normed space. Then:

(1) $\left(X,\left\{\mu^{(n)}\right\}, T\right)$ is called a matrix non-Archimedean random normed space if for each positive integer $n,\left(M_{n}(X),\left\{\mu^{(n)}\right\}, T\right)$ is a non-Archimedean random normed space and $\mu_{A x B}^{(k)}(t) \geq \mu_{x}^{(n)}\left(\frac{t}{\|A\| \cdot\|B\|}\right)$ for all $t>0, A \in M_{k, n}(\mathbb{R}), x=\left[x_{i j}\right] \in M_{n}(X)$ and $B \in M_{n, k}(\mathbb{R})$ with $\|A\| \cdot\|B\| \neq 0$.

(2) $\left(X,\left\{\mu^{(n)}\right\}, T\right)$ is called a matrix non-Archimedean random Banach space if $(X, \mu, T)$ is a non-Archimedean random Banach space and $\left(X,\left\{\mu^{(n)}\right\}, T\right)$ is a matrix nonArchimedean random normed space.

Definition 2.4 Let $\mathcal{E}$ be a set. A function $d: \mathcal{E} \times \mathcal{E} \rightarrow[0, \infty]$ is called a generalized metric on $\mathcal{E}$ if $d$ satisfies the following conditions:

(1) $d(x, y)=0$ if and only if $x=y$;

(2) $d(x, y)=d(y, x)$ for all $x, y \in \mathcal{E}$;

(3) $d(x, z) \leq d(x, y)+d(y, z)$ for all $x, y, z \in \mathcal{E}$.

We note that the only one difference of the generalized metric from the usual metric is that the range of the former is permitted to include infinity.

The following theorem is very useful for proving our main results; it is due to Diaz and Margolis [30].

Theorem 2.5 [30] Let $(\Omega, d)$ be a complete generalized metric space and let $\mathcal{J}: \Omega \rightarrow \Omega$ be a strictly contractive mapping with Lipschitz constant $L<1$. Then for each given element 
$x \in \Omega$, either

$$
d\left(\mathcal{J}^{n} x, \mathcal{J}^{n+1} x\right)=\infty
$$

for all non-negative integers $n$ or there exists a positive integer $n_{0}$ such that:

(1) $d\left(\mathcal{J}^{n} x, \mathcal{J}^{n+1} x\right)<\infty$ for all $n \geq n_{0}$;

(2) the sequence $\left\{\mathcal{J}^{n} x\right\}$ is convergent to a fixed point $x^{*}$ of $\mathcal{J}$;

(3) $x^{*}$ is the unique fixed point of $\mathcal{J}$ in the set

$$
\Delta:=\left\{y \in \Omega \mid d\left(\mathcal{J}^{n_{0}} x, y\right)<\infty\right\}
$$

(4) $d\left(y, x^{*}\right) \leq \frac{d(y, \mathcal{J} y)}{1-L}$, for all $y \in \Delta$.

Throughout this paper, we assume that $X$ be a normed space and $\left(Y, \mu^{(n)}, T\right)$ be a matrix non-Archimedean random Banach space.

We note that a mapping $r: X \rightarrow Y$ satisfies the functional equation (1.5) if and only if there exists a reciprocal mapping $r: X \rightarrow Y$ satisfying the reciprocal functional equation (1.1) [22].

For a mapping $r: X \rightarrow Y$, define $D_{m} r: X^{m} \rightarrow Y$ and $D_{m} r_{n}: M_{n}\left(X^{m}\right) \rightarrow M_{n}(Y)$ by

$$
\begin{aligned}
& D_{m} r\left(x_{1}, \ldots, x_{m}\right):= \frac{\prod_{\ell=2}^{m} r\left(x_{1}+x_{\ell}\right)}{\sum_{\ell=2}^{m}\left[\prod_{\substack{k=2 \\
k \neq \ell}}^{m} r\left(x_{1}+x_{k}\right)\right]} \\
&-\frac{\prod_{\ell=1}^{m} r\left(x_{\ell}\right)}{\sum_{\ell=2}^{m} r\left(x_{1}\right)\left[\prod_{\substack{k=2 \\
k \neq \ell}}^{m} r\left(x_{k}\right)\right]+(m-1) \prod_{\ell=2}^{m} r\left(x_{\ell}\right)}, \\
& D_{m} r_{n}\left(\left[x_{1 i j}\right],\left[x_{2 i j}\right], \ldots,\left[x_{m i j}\right]\right):= \frac{\prod_{\ell=2}^{m} r\left(\left[x_{1 i j}\right]+\left[x_{\ell i j}\right]\right)}{\sum_{\ell=2}^{m}\left[\prod_{\substack{k=2 \\
k \neq \ell}}^{m} r\left(\left[x_{1 i j}\right]+\left[x_{k i j}\right]\right)\right]} \\
&-\frac{\prod_{\ell=1}^{m} r\left(\left[x_{\ell i j}\right]\right)}{\sum_{\ell=2}^{m} r\left(\left[x_{1 i j}\right]\right)\left[\prod_{\substack{k=2 \\
k \neq \ell}}^{m} r\left(\left[x_{k i j}\right]\right)\right]+(m-1) \prod_{\ell=2}^{m} r\left(\left[x_{\ell i j}\right]\right)},
\end{aligned}
$$

for all $x_{1}, \ldots, x_{m} \in X$ and all $x_{1}^{\prime}=\left[x_{1 i j}\right], \ldots, x_{m}^{\prime}=\left[x_{m i j}\right] \in M_{n}(X)$.

\section{Generalized Hyers-Ulam stability of (1.5): direct method}

Theorem 3.1 Let $\varphi: X^{m} \rightarrow \mathcal{D}^{+}$be a function such that there exists $\alpha \in \mathbb{R}$ with $0<|\alpha|<|2|$ such that

$$
\varphi_{\frac{x_{1}}{2}, \ldots, \frac{x_{m}}{2}}(t) \geq \varphi_{x_{1}, \ldots, x_{m}}\left(\frac{t}{|\alpha|}\right)
$$

for all $x_{1}, \ldots, x_{m} \in X$ and all $t>0$ and $\lim _{l \rightarrow \infty} T_{\kappa=l}^{\infty}\left(\varphi_{\frac{x}{2^{k+1}}, \ldots, \frac{x}{2^{k+1}}}\left(\frac{|2|^{\kappa}}{|m-1|} t\right)\right)=1$ for all $x \in X$ and all $t>0$. If a function $f: X \rightarrow Y$ satisfies the functional inequality

$$
\mu_{D_{m f_{n}}\left(\left[x_{1 i j}\right], \ldots,\left[x_{m i j}\right]\right)}^{(n)}(t) \geq \sum_{i, j=1}^{n} \varphi_{x_{1 i j}, \ldots, x_{m i j}}(t)
$$


for all $x_{1}^{\prime}=\left[x_{1 i j}\right], \ldots, x_{m}^{\prime}=\left[x_{m i j}\right] \in M_{n}(X)$ and all $t>0$, then there exists a unique reciprocal mapping $r: X \rightarrow Y$ which satisfies (1.5) and the inequality

$$
\mu_{f_{n}\left(\left[x_{i j}\right]\right)-r_{n}\left(\left[x_{i j}\right]\right)}^{(n)}(t) \geq T\left(T_{l=0}^{\infty}\left(\varphi \frac{x_{i j}}{2^{l+1}, \ldots, x_{i j}}{\frac{2^{l+1}}{2}}\left(\frac{|2|^{l}}{|m-1| n^{2}} t\right)\right): i, j=1, \ldots, n\right)
$$

for all $x=\left[x_{i j}\right] \in M_{n}(X)$ and all $t>0$.

Proof Let $n=1$. Then (3.2) is equivalent to

$$
\mu_{D_{m} f\left(x_{1}, \ldots, x_{m}\right)}(t) \geq \varphi_{x_{1}, \ldots, x_{m}}(t)
$$

for all $x_{1}, \ldots, x_{m} \in X$ and all $t>0$. Replacing $x_{i}$ by $\frac{x}{2}$ for $i=1, \ldots, m$ in (3.4), we get

$$
\mu_{\frac{1}{m-1}\left[f(x)-\frac{1}{2} f\left(\frac{x}{2}\right)\right]}(t) \geq \varphi_{\frac{x}{2}, \ldots, \frac{x}{2}}(t)
$$

and so

$$
\mu_{f(x)-\frac{1}{2} f\left(\frac{x}{2}\right)}(t) \geq \varphi_{\frac{x}{2}, \ldots, \frac{x}{2}}\left(\frac{1}{|m-1|} t\right)
$$

for all $x \in X$ and all $t>0$. Now, replacing $x$ by $\frac{x}{2}$ in (3.5), we obtain

$$
\mu_{f\left(\frac{x}{2}\right)-\frac{1}{2} f\left(\frac{x}{2^{2}}\right)}(t) \geq \varphi_{\frac{x}{2^{2}}, \cdots, \frac{x}{2^{2}}}\left(\frac{1}{|m-1|} t\right)
$$

for all $x \in X$ and all $t>0$. By (3.5) and (3.6),

$$
\begin{aligned}
\mu_{f(x)-\frac{1}{2^{2}} f\left(\frac{x}{2^{2}}\right)}(t) & \geq T\left(\mu_{f(x)-\frac{1}{2} f\left(\frac{x}{2}\right)}(t), \mu_{\frac{1}{2} f\left(\frac{x}{2}\right)-\frac{1}{2^{2}} f\left(\frac{x}{2^{2}}\right)}(t)\right) \\
& =T\left(\mu_{f(x)-\frac{1}{2} f\left(\frac{x}{2}\right)}(t), \mu_{f\left(\frac{x}{2}\right)-\frac{1}{2} f\left(\frac{x}{2^{2}}\right)}(|2| t)\right) \\
& \geq T\left(\varphi_{\frac{x}{2}, \ldots, \frac{x}{2}}\left(\frac{1}{|m-1|} t\right), \varphi_{\frac{x}{2^{2}}, \ldots, \frac{x}{2^{2}}}\left(\frac{|2|}{|m-1|} t\right)\right)
\end{aligned}
$$

for all $x \in X$ and all $t>0$. Proceeding and using induction arguments on a positive integer $J$, we arrive at

$$
\mu_{f(x)-\frac{1}{2 J} f\left(\frac{x}{2 J}\right)}(t) \geq T_{l=0}^{J-1}\left(\varphi_{\frac{x}{2^{l+1}}, \ldots, \frac{x}{2^{l+1}}}\left(\frac{|2|^{l}}{|m-1|} t\right)\right)
$$

for all $x \in X$ and all $t>0$. For any positive integer $t, x \in X$ and $t>0$, we have

$$
\mu \frac{1}{2^{l+1} f\left(\frac{x}{2^{l+1}}\right)-\frac{1}{2^{f}} f\left(\frac{x}{2^{t}}\right)}(t)=\mu_{\frac{1}{2^{l}}\left[f\left(\frac{x}{2^{t}}\right)-\frac{1}{2} f\left(\frac{x}{2^{l+1}}\right)\right]}(t) \geq \varphi_{\frac{x}{2^{l+1}}, \ldots, \frac{x}{2^{l+1}}}\left(\frac{|2|^{l}}{|m-1|} t\right) .
$$

Hence,

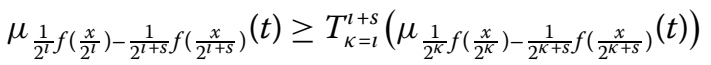

$$
\begin{aligned}
& \geq T_{\kappa=l}^{l+s}\left(\varphi_{\frac{x}{2^{\kappa+1}}, \ldots, \frac{x}{2^{\kappa+1}}}\left(\frac{|2|^{\kappa}}{|m-1|} t\right)\right)
\end{aligned}
$$


for all $x \in X$, all $t>0$ and $\imath \geq 0$. Since $\lim _{l \rightarrow \infty} T_{\kappa=l}^{\infty}\left(\varphi_{\frac{x}{2^{\kappa+1}}, \ldots, \frac{x}{2^{\kappa+1}}}\left(\frac{|2|^{\kappa}}{|m-1|} t\right)\right)=1$ for all $x \in X$ and all $t>0$, it follows that the sequence $\left\{\frac{1}{2^{J}} f\left(\frac{x}{2^{j}}\right)\right\}$ is a Cauchy sequence in the matrix nonArchimedean random Banach space $\left(Y, \mu^{(n)}, T\right)$. Hence, we can define a mapping $r: X \rightarrow$ $Y$ by

$$
\lim _{J \rightarrow \infty} \mu_{\frac{1}{2^{J}} f\left(\frac{x}{2^{J}}\right)-r(x)}(t)=1
$$

for all $x \in X$ and all $t>0$.

Moreover, letting $\mathrm{J} \rightarrow \infty$ in (3.7), we get

$$
\mu_{f(x)-r(x)}(t) \geq T_{l=0}^{\infty}\left(\varphi \frac{x}{2^{l+1}, \ldots, \frac{x}{2^{l+1}}}\left(\frac{|2|^{l}}{|m-1|} t\right)\right)
$$

for all $x \in X$ and all $t>0$. To show that $r$ satisfies (1.5), replacing $\left(x_{1}, \ldots, x_{m}\right)$ by $\left(\frac{x_{1}}{2 \jmath}, \ldots, \frac{x_{m}}{2 \jmath}\right)$ in (3.4) and using (3.1), we obtain

$$
\mu_{\frac{1}{2 J} D_{m} f\left(\frac{x_{1}}{2 J}, \ldots, \frac{x_{m}}{2 J}\right)}(t) \geq \varphi_{\frac{x_{1}}{2 J}, \ldots, \frac{x_{m}}{2 J}}\left(|2|^{J} t\right) \geq \varphi_{x_{1}, \ldots, x_{m}}\left(\frac{|2|^{J}}{|\alpha|^{J}} t\right)
$$

for all $x_{1}, \ldots, x_{m} \in X$, for all positive integer $J$ and all $t>0$. Since $\lim _{J \rightarrow \infty} \varphi_{x_{1}, \ldots, x_{m}}\left(\left(\frac{|2|}{|\alpha|}\right)^{\jmath} t\right)=1$, we see that $r$ satisfies (1.5), for all $x_{1}, \ldots, x_{m} \in X$ and all $t>0$.

We note that $e_{j} \in M_{1, n}(\mathbb{R})$ means that $j$ th component is 1 and the others are zero, $E_{i j} \in$ $M_{n}(\mathbb{R})$ means that $(i, j)$-component is 1 and the others are zero, and $E_{i j} \otimes x \in M_{n}(X)$ means that $(i, j)$-component is $x$ and the others are zero. Since $\mu_{E_{p q} \otimes x}^{(n)}(t)=\mu_{x}(t)$, we have

$$
\mu_{\left[x_{i j}\right]}^{(n)}(t)=\mu_{\sum_{i, j=1}^{n} E_{i j} \otimes x_{i j}}^{(n)}(t) \geq T\left(\mu_{E_{i j} \otimes x_{i j}}^{(n)}\left(t_{i j}\right): i, j=1,2, \ldots, n\right)=T\left(\mu_{x_{i j}}\left(t_{i j}\right): i, j=1,2, \ldots, n\right),
$$

where $t=\sum_{i, j=1}^{n} t_{i j}$. So $\mu_{\left[x_{i j}\right]}^{(n)}(t) \geq T\left(\mu_{x_{i j}}\left(\frac{t}{n^{2}}\right): i, j=1,2, \ldots, n\right)$.

By (3.10),

$$
\begin{aligned}
\mu_{f_{n}\left(\left[x_{i j}\right]\right)-r_{n}\left(\left[x_{i j}\right]\right)}^{(n)}(t) & \geq T\left(\mu_{f\left(x_{i j}\right)-r\left(x_{i j}\right)}\left(\frac{t}{n^{2}}\right): i, j=1, \ldots, n\right) \\
& \geq T\left(T_{l=0}^{\infty}\left(\varphi \frac{x_{i j}}{2^{l+1}, \ldots, \frac{x_{i j}}{2^{l+1}}}\left(\frac{|2|^{l}}{|m-1| n^{2}} t\right)\right): i, j=1, \ldots, n\right)
\end{aligned}
$$

for all $x=\left[x_{i j}\right] \in M_{n}(X)$ and all $t>0$. Thus $r: X \rightarrow Y$ is a unique mapping satisfying (3.3), as desired.

Theorem 3.2 Let $\varphi: X^{m} \rightarrow \mathcal{D}^{+}$be a function such that there exists $\alpha \in \mathbb{R}$ with $0<|2|<|\alpha|$ such that

$$
\varphi_{2 x_{1}, \ldots, 2 x_{m}}(t) \geq \varphi_{x_{1}, \ldots, x_{m}}(|\alpha| t)
$$

for all $x_{1}, \ldots, x_{m} \in X$ and all $t>0$ and $\lim _{l \rightarrow \infty} T_{\kappa=l}^{\infty}\left(\varphi_{2^{\kappa} x, \ldots, 2^{\kappa} x}\left(\frac{t}{|m-1||2|^{\kappa+1}}\right)\right)=1$ for all $x \in X$ and all $t>0$. If a function $f: X \rightarrow Y$ satisfies the functional inequality

$$
\mu_{D_{m} f_{n}\left(\left[x_{1 i j}\right], \ldots,\left[x_{m i j}\right]\right)}^{(n)}(t) \geq \sum_{i, j=1}^{n} \varphi_{x_{1 i j}, \ldots, x_{m i j}}(t)
$$


for all $x_{1}^{\prime}=\left[x_{1 i j}\right], \ldots, x_{m}^{\prime}=\left[x_{m i j}\right] \in M_{n}(X)$ and all $t>0$, then there exists a unique reciprocal mapping $r: X \rightarrow Y$ which satisfies (1.5) and the inequality

$$
\mu_{f_{n}\left(\left[x_{i j]}\right]\right)-r_{n}\left(\left[x_{i j}\right]\right)}^{(n)}(t) \geq T\left(T_{l=0}^{\infty}\left(\varphi_{2^{l} x_{i j}, \ldots, 2^{l} x_{i j}}\left(\frac{t}{n^{2}|m-1||2|^{i+1}}\right)\right): i, j=1, \ldots, n\right)
$$

for all $x=\left[x_{i j}\right] \in M_{n}(X)$ and all $t>0$.

Proof Let $n=1$. Then (3.13) is equivalent to

$$
\mu_{D_{m} f\left(x_{1}, \ldots, x_{m}\right)}(t) \geq \varphi_{x_{1}, \ldots, x_{m}}(t)
$$

for all $x_{1}, \ldots, x_{m} \in X$ and all $t>0$. The rest of the proof is obtained by replacing $x_{i}$ by $x$ for $i=1,2, \ldots, m$ in (3.15) and proceeding further by similar arguments as in Theorem 3.1.

Corollary 3.3 Let $\gamma \in\{-1,1\}$ be fixed. Let $f: X \rightarrow Y$ be a mapping and let there exist real numbers $q \neq-1$ and $\theta \geq 0$ with $-\gamma<q \gamma$ such that

$$
\mu_{D_{m f_{n}}\left(\left[x_{1 i j}\right], \ldots,\left[x_{m i j}\right]\right)}^{(n)}(t) \geq \frac{t}{t+\sum_{i, j=1}^{n} \theta\left(\left\|x_{1 i j}\right\|^{q}+\cdots+\left\|x_{m i j}\right\|^{q}\right)}
$$

for all $x_{1}^{\prime}=\left[x_{1 i j}\right], \ldots, x_{m}^{\prime}=\left[x_{m i j}\right] \in M_{n}(X)$ and all $t>0$. If

$$
\lim _{l \rightarrow \infty} T_{\kappa=l}^{\infty}\left(\frac{t}{t+\theta m|m-1|\|x\|^{q}\left(|2|^{\kappa q+q+\kappa}\right)^{\frac{-\gamma-1}{2}}\left(|2|^{\kappa q+\kappa+1}\right)^{\frac{-\gamma+1}{2}}}\right)=1
$$

for all $x \in X$ and all $t>0$, then there exists a unique reciprocal mapping $r: X \rightarrow Y$ such that

$$
\mu_{f_{n}\left(\left[x_{i j}\right]\right)-r_{n}\left(\left[x_{i j}\right]\right)}^{(n)}(t) \geq \begin{cases}T\left(T_{l=0}^{\infty}\left(\frac{t}{t+\frac{\left.\theta m n^{2}|m-1||| x_{i j}\right|^{q}}{|2|^{\mid q+q+1}}}\right): i, j=1,2, \ldots, n\right) & \text { for } \gamma=1, \\ T\left(T_{l=0}^{\infty}\left(\frac{t}{t+\left.\theta m n^{2}|m-1||| x_{i j}\left|\|^{q}\right| 2\right|^{\mid q+l+1}}\right): i, j=1,2, \ldots, n\right) & \text { for } \gamma=-1\end{cases}
$$

for all $x=\left[x_{i j}\right] \in M_{n}(X)$ and all $t>0$.

Proof If we choose $\varphi_{x_{1}, \ldots, x_{m}}(t)=\frac{t}{t+\theta\left(\left\|x_{1}\right\|^{q}+\cdots+\left\|x_{m}\right\|^{q)}\right.}$, for all $t>0$ and all $x_{1}, \ldots, x_{m} \in X$, then by Theorem 3.1, we arrive at

$$
\mu_{f_{n}\left(\left[x_{i j}\right]\right)-r_{n}\left(\left[x_{i j}\right]\right)}^{(n)}(t) \geq T\left(T_{l=0}^{\infty}\left(\frac{t}{t+\frac{\left.\theta m n^{2}|m-1||| x_{i j}\right|^{q}}{|2|^{\mid q+q+1}}}\right): i, j=1,2, \ldots, n\right)
$$

for all $x=\left[x_{i j}\right] \in M_{n}(X)$, all $t>0$ and $\gamma=1$, and using Theorem 3.2, we arrive at

$$
\mu_{f_{n}\left(\left[x_{i j}\right]\right)-r_{n}\left(\left[x_{i j}\right]\right)}^{(n)}(t) \geq T\left(T_{i=0}^{\infty}\left(\frac{t}{t+\theta m n^{2}|m-1|\left\|x_{i j}\right\|^{q}|2|^{\mid q+l+1}}\right): i, j=1,2, \ldots, n\right)
$$

for all $x=\left[x_{i j}\right] \in M_{n}(X)$, all $t>0$ and $\gamma=-1$. 


\section{Generalized Hyers-Ulam stability of equation (1.5): fixed point method}

Theorem 4.1 Suppose that the mapping $f: X \rightarrow Y$ satisfies the inequality

$$
\mu_{D_{m} f_{n}\left(\left[x_{1 i j}\right], \ldots,\left[x_{m i j}\right]\right)}^{(n)}(t) \geq \sum_{i, j=1}^{n} \phi_{x_{1 i j}, \ldots, x_{m i j}}(t)
$$

for all $x_{1}^{\prime}=\left[x_{1 i j}\right], \ldots, x_{m}^{\prime}=\left[x_{m i j}\right] \in M_{n}(X)$ and all $t>0$, where $\phi: X^{m} \rightarrow \mathcal{D}^{+}$is a given function. If there exists $L<1$ such that

$$
\phi_{\frac{x_{1}}{2}, \ldots, \frac{x_{m}}{2}}(t) \geq \phi_{x_{1}, \ldots, x_{m}}\left(\frac{1}{L|2|} t\right)
$$

for all $x_{1}, \ldots, x_{m} \in X$ and all $t>0$, then there exists a unique reciprocal mapping $r: X \rightarrow Y$ which satisfies (1.5) and the inequality

$$
\mu_{f_{n}\left(\left[x_{i j}\right]\right)-r_{n}\left(\left[x_{i j}\right]\right)}^{(n)}(t) \geq T\left(\phi_{x_{i j}, \ldots, x_{i j}}\left(\frac{1-L}{n^{2} L|2||m-1|} t\right): i, j=1, \ldots, n\right)
$$

for all $x=\left[x_{i j}\right] \in M_{n}(X)$ and all $t>0$.

Proof Let $n=1$. Then (4.1) is equivalent to

$$
\mu_{D_{m} f\left(x_{1}, \ldots, x_{m}\right)}(t) \geq \phi_{x_{1}, \ldots, x_{m}}(t)
$$

for all $x_{1}, \ldots, x_{m} \in X$ and all $t>0$.

Define a set $\mathcal{S}$ by

$$
\mathcal{S}=\{h: X \rightarrow Y \mid h \text { is a function }\}
$$

and introduce the generalized metric $d$ on $\mathcal{S}$ as follows:

$$
d(g, h)=\inf \left\{\lambda \in \mathbb{R}_{+}: \mu_{g(x)-h(x)}(\lambda t) \geq \phi_{x, \ldots, x}(t), \forall x \in X, \forall t>0\right\},
$$

where, as usual, $\inf \emptyset=+\infty$. It is easy to show that $(\mathcal{S}, d)$ is complete (see [27], Lemma 2.1).

Define a mapping $\sigma: \mathcal{S} \rightarrow \mathcal{S}$ by

$$
\sigma h(x)=\frac{1}{2} h\left(\frac{x}{2}\right) \quad(x \in X)
$$

for all $h \in \mathcal{S}$. We claim that $\sigma$ is strictly contractive on $\mathcal{S}$. For any given $g, h \in \mathcal{S}$, let $\epsilon_{g h} \in$ $[0, \infty]$ be an arbitrary constant with $d(g, h) \leq \epsilon_{g h}$. Hence

$$
\begin{aligned}
d(g, h) \leq \epsilon_{g h} & \Rightarrow \quad \mu_{g(x)-h(x)}\left(\epsilon_{g h} t\right) \geq \phi_{x, \ldots, x}(t), \quad \forall x \in X, \forall t>0 \\
& \Rightarrow \quad \mu_{\frac{1}{2} g\left(\frac{x}{2}\right)-\frac{1}{2} h\left(\frac{x}{2}\right)}\left(\epsilon_{g h} t\right) \geq \phi_{\frac{x}{2}, \ldots, \frac{x}{2}}(|2| t), \quad \forall x \in X, \forall t>0 \\
& \Rightarrow \quad \mu_{\frac{1}{2} g\left(\frac{x}{2}\right)-\frac{1}{2} h\left(\frac{x}{2}\right)}\left(\epsilon_{g h} t\right) \geq \phi_{x, \ldots, x}\left(\frac{1}{L} t\right), \quad \forall x \in X, \forall t>0 \\
& \Rightarrow \quad \mu_{\frac{1}{2} g\left(\frac{x}{2}\right)-\frac{1}{2} h\left(\frac{x}{2}\right)}\left(L \epsilon_{g h} t\right) \geq \phi_{x, \ldots, x}(t), \quad \forall x \in X, \forall t>0 \\
& \Rightarrow \quad d(\sigma g, \sigma h) \leq L \epsilon_{g h} .
\end{aligned}
$$


Therefore, we see that

$$
d(\sigma g, \sigma h) \leq L d(g, h), \quad \text { for all } g, h \in \mathcal{S}
$$

that is, $\sigma$ is strictly contractive mapping of $\mathcal{S}$, with the Lipschitz constant $L<1$.

Now, replacing $x_{i}$ by $\frac{x}{2}$ for $i=1, \ldots, m$ in (4.4), we get

$$
\mu_{f(x)-\frac{1}{2} f\left(\frac{x}{2}\right)}(t) \geq \phi_{\frac{x}{2}, \ldots, \frac{x}{2}}\left(\frac{1}{|m-1|} t\right) \geq \phi_{x, \ldots, x}\left(\frac{1}{L|2||m-1|} t\right)
$$

for all $x \in X$ and all $t>0$. Hence (4.5) implies that $d(f, \sigma f) \leq L|2||m-1|<\infty$. Hence by applying the fixed point alternative Theorem 2.5, there exists a function $r: X \rightarrow Y$ satisfying the following:

(1) $r$ is a fixed point of $\sigma$, that is,

$$
r(x)=\frac{1}{2} r\left(\frac{x}{2}\right)
$$

for all $x \in X$. The mapping $r$ is the unique fixed point of $\sigma$ in the set

$$
\mathcal{M}=\{g \in \mathcal{S} \mid d(f, g)<\infty\} .
$$

This implies that $r$ is the unique mapping satisfying (4.7) such that there exists $\epsilon \epsilon$ $(0, \infty)$ satisfying

$$
\mu_{f(x)-r(x)}(\epsilon t) \geq \phi_{x, \ldots, x}(t), \quad \forall x \in X, \forall t>0
$$

(2) $d\left(\sigma^{n} f, r\right) \rightarrow 0$ as $n \rightarrow \infty$. Thus we have

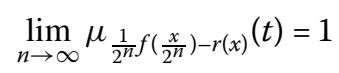

for all $x \in X$ and all $t>0$.

(3) $d(r, f) \leq \frac{1}{1-L} d(f, \sigma f)$ which implies

$$
d(r, f) \leq \frac{L|2||m-1|}{1-L}
$$

From (4.2), (4.4), and (4.8), we have

$$
\mu_{\frac{1}{2^{n}} D_{m} f\left(\frac{x_{1}}{2^{n}}, \ldots, \frac{x_{m}}{2^{n}}\right)}(t) \geq \phi_{\frac{x_{1}}{2^{n}}, \ldots, \frac{x_{m}}{2^{n}}}\left(|2|^{n} t\right) \geq \phi_{x_{1}, \ldots, x_{m}}\left(\left(\frac{1}{L}\right)^{n} t\right)
$$

for all $x_{1}, \ldots, x_{m} \in X$ and all $t>0$. Since $\lim _{n \rightarrow \infty} \phi_{x_{1}, \ldots, x_{m}}\left(\left(\frac{1}{L}\right)^{n} t\right)=1, r$ satisfies (1.5).

We note that $e_{j} \in M_{1, n}(\mathbb{R})$ means that the $j$ th component is 1 and the others are zero, $E_{i j} \in$ $M_{n}(\mathbb{R})$ means that the $(i, j)$-component is 1 and the others are zero, and $E_{i j} \otimes x \in M_{n}(X)$ means that the $(i, j)$-component is $x$ and the others are zero. Since $\mu_{E_{p q} \otimes x}^{(n)}(t)=\mu_{x}(t)$, we have

$$
\mu_{\left[x_{i j}\right]}^{(n)}(t)=\mu_{\sum_{i, j=1}^{n} E_{i j} \otimes x_{i j}}^{(n)}(t) \geq T\left(\mu_{E_{i j} \otimes x_{i j}}^{(n)}\left(t_{i j}\right): i, j=1,2, \ldots, n\right)=T\left(\mu_{x_{i j}}\left(t_{i j}\right): i, j=1,2, \ldots, n\right),
$$

where $t=\sum_{i, j=1}^{n} t_{i j}$. So $\mu_{\left[x_{i j}\right]}^{(n)}(t) \geq T\left(\mu_{x_{i j}}\left(\frac{t}{n^{2}}\right): i, j=1,2, \ldots, n\right)$. 
By (4.9),

$$
\begin{aligned}
\mu_{f_{n}\left(\left[x_{i j}\right]\right)-r_{n}\left(\left[x_{i j}\right]\right)}^{(n)}(t) & \geq T\left(\mu_{f\left(x_{i j}\right)-r\left(x_{i j}\right)}\left(\frac{t}{n^{2}}\right): i, j=1, \ldots, n\right) \\
& \geq T\left(\phi_{x_{i i j}, \ldots, x_{i j}}\left(\frac{1-L}{n^{2} L|2||m-1|} t\right): i, j=1, \ldots, n\right)
\end{aligned}
$$

for all $x=\left[x_{i j}\right] \in M_{n}(X)$ and all $t>0$. Thus $r: X \rightarrow Y$ is a unique reciprocal mapping satisfying (4.3).

Theorem 4.2 Suppose that the mapping $f: X \rightarrow Y$ satisfies the inequality

$$
\mu_{D_{m f}\left(\left[x_{1 i j}\right], \ldots,\left[x_{m i j}\right]\right)}^{(n)}(t) \geq \sum_{i, j=1}^{n} \phi_{x_{1 i j}, \ldots, x_{m i j}}(t)
$$

for all $x_{1}^{\prime}=\left[x_{1 i j}\right], \ldots, x_{m}^{\prime}=\left[x_{m i j}\right] \in M_{n}(X)$ and all $t>0$, where $\phi: X^{m} \rightarrow \mathcal{D}^{+}$is a given function. If there exists $L<1$ such that

$$
\phi_{2 x_{1}, \ldots, 2 x_{m}}(t) \geq \phi_{x_{1}, \ldots, x_{m}}\left(\frac{|2|}{L} t\right)
$$

for all $x_{1}, \ldots, x_{m} \in X$ and all $t>0$, then there exists a unique reciprocal mapping $r: X \rightarrow Y$ which satisfies (1.5) and the inequality

$$
\mu_{f_{n}\left(\left[x_{i j}\right]\right)-r_{n}\left(\left[x_{i j}\right]\right)}^{(n)}(t) \geq T\left(\phi_{x_{i j}, \ldots, x_{i j}}\left(\frac{1-L}{n^{2}|2||m-1|} t\right): i, j=1, \ldots, n\right)
$$

for all $x=\left[x_{i j}\right] \in M_{n}(X)$ and all $t>0$.

Proof The proof is similar to the proof of Theorem 4.1.

Corollary 4.3 Let $\gamma \in\{-1,1\}$ be fixed. Let $f: X \rightarrow Y$ be a mapping and let there exist real numbers $q \neq-1$ and $\theta \geq 0$ with $-\gamma<q \gamma$ such that

$$
\mu_{D_{m f}\left(\left[x_{1 i j}\right], \ldots,\left[x_{m i j}\right]\right)}^{(n)}(t) \geq \frac{t}{t+\sum_{i, j=1}^{n} \theta\left(\left\|x_{1 i j}\right\|^{q}+\cdots+\left\|x_{m i j}\right\|^{q}\right)}
$$

for all $x_{1}^{\prime}=\left[x_{1 i j}\right], \ldots, x_{m}^{\prime}=\left[x_{m i j}\right] \in M_{n}(X)$ and all $t>0$. Then there exists a unique reciprocal mapping $r: X \rightarrow Y$ such that

$$
\mu_{f_{n}\left(\left[x_{i j}\right]\right)-r_{n}\left(\left[x_{i j}\right]\right)}^{(n)}(t) \geq \begin{cases}T\left(\frac{\left(|2|^{q+1}-1\right) t}{\left(|2|^{q+1}-1\right) t+\theta m n^{2}|2||m-1||| x_{i j} \|^{q}}: i, j=1,2, \ldots, n\right) & \text { for } \gamma=1, \\ T\left(\frac{\left(1-|2|^{q+1}\right) t}{\left(1-|2|^{\mid q+1}\right) t+\theta m n^{2}|2||m-1||| x_{i j} \|^{q}}: i, j=1,2, \ldots, n\right) & \text { for } \gamma=-1\end{cases}
$$

for all $x=\left[x_{i j}\right] \in M_{n}(X)$ and all $t>0$.

Proof If we choose $\phi_{x_{1}, \ldots, x_{m}}(t)=\frac{t}{t+\theta\left(\left\|x_{1}\right\|\left\|^{q}+\cdots+\right\| x_{m} \|^{q}\right)}$, for all $t>0$ and all $x_{1}, \ldots, x_{m} \in X$, then by Theorem 4.1, we arrive at

$$
\mu_{f_{n}\left(\left[x_{i j}\right]\right)-r_{n}\left(\left[x_{i j}\right]\right)}^{(n)}(t) \geq T\left(\frac{\left(|2|^{q+1}-1\right) t}{\left(|2|^{q+1}-1\right) t+\theta m n^{2}|2||m-1|\left\|x_{i j}\right\|^{q}}: i, j=1,2, \ldots, n\right)
$$


for all $x=\left[x_{i j}\right] \in M_{n}(X)$, all $t>0, \gamma=1, L=|2|^{-q-1}$, and using Theorem 4.2, we arrive at

$$
\mu_{f_{n}\left(\left[x_{i j}\right]\right)-r_{n}\left(\left[x_{i j}\right]\right)}^{(n)}(t) \geq T\left(\frac{\left(1-|2|^{q+1}\right) t}{\left(1-|2|^{q+1}\right) t+\theta m n^{2}|2||m-1|\left\|x_{i j}\right\|^{q}}: i, j=1,2, \ldots, n\right)
$$

for all $x=\left[x_{i j}\right] \in M_{n}(X)$, all $t>0, \gamma=-1$ and $L=|2|^{q+1}$.

\section{Competing interests}

The authors declare that they have no competing interests.

\section{Authors' contributions}

All authors contributed equally in drafting this manuscript and giving the main proofs. All authors read and approved the final manuscript.

\section{Author details}

'Department of Mathematics, Urmia University, P.O. Box 165, Urmia, Iran. ${ }^{2}$ Research Institute for Natural Sciences, Hanyang University, Seoul, 133-791, Republic of Korea.

\section{Acknowledgements}

C Park was supported by the Basic Science Research Program through the National Research Foundation of Korea funded by the Ministry of Education, Science and Technology.

Received: 17 May 2015 Accepted: 30 September 2015 Published online: 09 October 2015

\section{References}

1. Ulam, SM: A Collection of the Mathematical Problems. Interscience, New York (1960)

2. Hyers, DH: On the stability of the linear functional equation. Proc. Natl. Acad. Sci. 27, $222-224$ (1941)

3. Aoki, T: On the stability of the linear transformation in Banach spaces. J. Math. Soc. Jpn. 2, $64-66$ (1950)

4. Rassias, TM: On the stability of the linear mapping in Banach spaces. Proc. Am. Math. Soc. 72, 297-300 (1978)

5. Rassias, JM: On approximation of approximately linear mappings by linear mappings. J. Funct. Anal. 46, 126-130 (1982)

6. Rassias, JM: On approximation of approximately linear mappings by linear mappings. Bull. Sci. Math. 108, 445-446 (1984)

7. Rassias, JM: Solution of a problem of Ulam. J. Approx. Theory 57, 268-273 (1989)

8. Găvruta, P: A generalization of the Hyers-Ulam-Rassias stability of approximately additive mappings. J. Math. Anal. Appl. 184, 431-436 (1994)

9. Cho, YJ, Eshaghi Gordji, M, Zolfaghari, S: Solutions and stability of generalized mixed type QC functional equations in random normed spaces. J. Inequal. Appl. 2010, Article ID 403101 (2010). doi:10.1155/2010/403101

10. Cholewa, PW: Remarks on the stability of functional equations. Aequ. Math. 27, 76-86 (1984)

11. Ebadian, A, Zolfaghari, S: Stability of a mixed additive and cubic functional equation in several variables in non-Archimedean spaces. Ann. Univ. Ferrara 58, 291-306 (2012)

12. Eshaghi Gordji, M, Ebadian, A, Zolfaghari, S: Stability of a functional equation deriving from cubic and quartic functions. Abstr. Appl. Anal. 2008, Article ID 801904 (2008)

13. Eshaghi Gordji, M, Kaboli-Gharetapeh, S, Park, C, Zolfaghari, S: Stability of an additive-cubic-quartic functional equation. Adv. Differ. Equ. 2009, Article ID 395693 (2009)

14. Eshaghi Gordji, M, Kaboli-Gharetapeh, S, Rassias, JM, Zolfaghari, S: Solution and stability of a mixed type additive, quadratic and cubic functional equation. Adv. Differ. Equ. 2009. Article ID 826130 (2009). doi:10.1155/2009/826130

15. Eshaghi Gordji, M, Moslehian, MS, Kaboli-Gharetapeh, S, Zolfaghari, S: Stability of a mixed type additive, quadratic, cubic and quartic functional equation. In: Khan, A, et al. (eds.) Nonlinear Analysis and Variational Problems, pp. 65-80. Springer, Berlin (2009)

16. Eshaghi Gordji, M, Zolfaghari, S, Rassias, JM, Bavand-Savadkouhi, M: Solution and stability of a mixed type cubic and quartic functional equation in quasi-Banach spaces. Abstr. Appl. Anal. 2009, Article ID 417473 (2009). doi:10.1155/2009/417473

17. Zolfaghari, S: Stability of generalized QCA-functional equation in p-Banach spaces. Int. J. Nonlinear Anal. Appl. 1 84-99 (2010)

18. Zolfaghari, S, Ebadian, A, Ostadbashi, S, De La Sen, M, Eshaghi Gordji, M: A fixed point approach to the Hyers-Ulam stability of an AQ functional equation in probabilistic modular spaces. Int. J. Nonlinear Anal. Appl. 4(2), 1-14 (2013)

19. Ravi, K, Senthil Kumar, BV: Ulam-Găvruta-Rassias stability of Rassias reciprocal functional equation. Glob. J. Appl. Math. Math. Sci. 3, 57-79 (2010)

20. Ravi, K, Rassias, JM, Senthil Kumar, BV: Ulam stability of reciprocal difference and adjoint functional equations. Aust. J. Math. Anal. Appl. 8(1), 13 (2011)

21. Ravi, K, Rassias, JM, Senthil Kumar, BV: Ulam stability of generalized reciprocal functional equation in several variables. Int. J. Appl. Math. Stat. 19, 1-19 (2010)

22. Ravi, K, Thandapani, E, Senthil Kumar, BV: Solution and stability of a reciprocal type functional equation in several variables. J. Nonlinear Sci. Appl. 7, 18-27 (2014)

23. Ravi, K, Rassias, JM, Senthil Kumar, BV: Generalized Hyers-Ulam stability and examples of non-stability of reciprocal type functional equation in several variables. Indian J. Sci. 2(3), 13-22 (2013)

24. Ravi, K, Rassias, JM, Senthil Kumar, BV: Example for the stability of reciprocal type functional equation controlled by product of different powers in singular case. Adv. Pure Math. 61, 16821-16825 (2013) 
25. Hensel, K: Über eine neue Begrundung der Theorie der algebraischen Zahlen. Jahresber. Dtsch. Math.-Ver. 6, 83-88 (1897)

26. Chang, SS, Cho, YJ, Kang, SM: Nonlinear Operator Theory in Probabilistic Metric Spaces. Nova Science Publishers, New York (2001)

27. Mihet, D, Radu, D: On the stability of the additive Cauchy functional equation in random normed spaces. J. Math. Anal. Appl. 343(1), 567-572 (2008)

28. Šerstnev, AN: On the notion of a random normed space. Dokl. Akad. Nauk SSSR 149(2), 280-283 (1963) (in Russian)

29. Schweizer, B, Sklar, A: Probabilistic Metric Spaces. North-Holland, New York (1983)

30. Diaz, JB, Margolis, B: A fixed point theorem of the alternative for the contractions on generalized complete metric space. Bull. Am. Math. Soc. 74, 305-309 (1968)

Submit your manuscript to a SpringerOpen ${ }^{\circ}$ journal and benefit from:

- Convenient online submission

Rigorous peer review

- Immediate publication on acceptance

- Open access: articles freely available online

- High visibility within the field

- Retaining the copyright to your article 